\title{
Études récentes sur des siphons et appareils dérivés
}

\section{Recent investigations on siphons and derived equipment}

\author{
PAR Y. PONSAR * \\ English synopsis, p. 112
}

\section{I. - LE REGULATEUR HYDROPNEUMATIQUE}

Ce régulateur de débit liquide contrôle toute une gamme de débits constants lors de leur pas. sage d'un bassin ou d'un canal dans un autre. La valeur de ces débits peut varier à volonté à I'aide d'un réglage direct à main ou d'une télécommande facile à établir. Cette valeur est indépendante des niveaux amont et aval.

La perte de charge minimum demandée par l'appareil peut être abaissée jusqu'aux environs de 4 ou $5 \mathrm{~cm}$ en cas de besoin.

L'appareil comprend essentiellement les pièces suivantes :

- Un déversoir surmonté d'une hotte étanche (fig. 1) dans laquelle règne une certaine dépression. Le plan d'eau à l'amont du déversoir est. calme en raison de la grande section de la branche amont et son niveau est $n_{1}$ que l'on cherche à régler. L'eau s'écoule par la branche aval de section plus petite où a lieu la dissipation d'énergie excédentaire sous forme de chute; cette dernière provoque un entraînement continu d'une partie de l'air emprisonné, ce qui tend à augmenter la dépression intérieure et à faire monter le niveau $n_{1}$.

- Un robinet d'air particulier, à commande

* M. Y. Ponsar est gérant de la Société Hydraulique et Urbanisme, 101-103, rue Victor-Hugo, à Bois-Colombes. Tél. CHA. 34-97.

Les appareils décrits font l'objet de demandes de brevets en France et à l'étranger; le premier est connu sous le nom de «Module Polhydra». pneumatique destiné à introduire de l'air dans la hotte pour réduire la dépression et abaisser le niveau $n_{1}$, ou simplement pour remplacer l'air évacué à l'extérieur et maintenir dans ce cas le niveau réglé à une valeur constante.

- Un équipage mobile appelé régleur. Cel équipage comporte Iui-même (fig. 2) : un flotteur détectant le niveau réglé, un tiroir de distribution d'air déprimé commandant les mouvements du robinet ci-dessus, enfin un dispositif destinc à provoquer l'équilibre de l'ensemble pour un niveau réglé à la hauteur voulue. Sur la figure 2, cette variation d'équilibre est obtenue par un lestage hydraulique du flotteur à l'aide des tubulures souples $t_{1} \mathrm{dl} t_{2}$.

Il est probablement inutile d'insister sur le fonctionnement : le niveau intérieur tend à monter en raison de l'air entraîné à l'extérieur, mais peut être abaissé ou maintenu à toute hauteur voulue $n_{1}$ par introduction d'air. On remarquera que si le niveau amont extérieur $N_{1}$ s'abaisse, la dépression intérieure augmente d'autant; en même temps, le niveau aval intérieur $n_{2}$ s'élève d'autant.

Basé sur un principe simple, cet appareil demande cependant les soins suivants lors de sa réalisation :

- Tout d'abord la hotte doit être suffisamment étanche à l'air et le rester. Si un dessin particulier de la branche aval est susceptible d'améliorer l'évacuation de l'air, l'étanchéité doit être cependant recherchée, ne sernit-ce que pour 


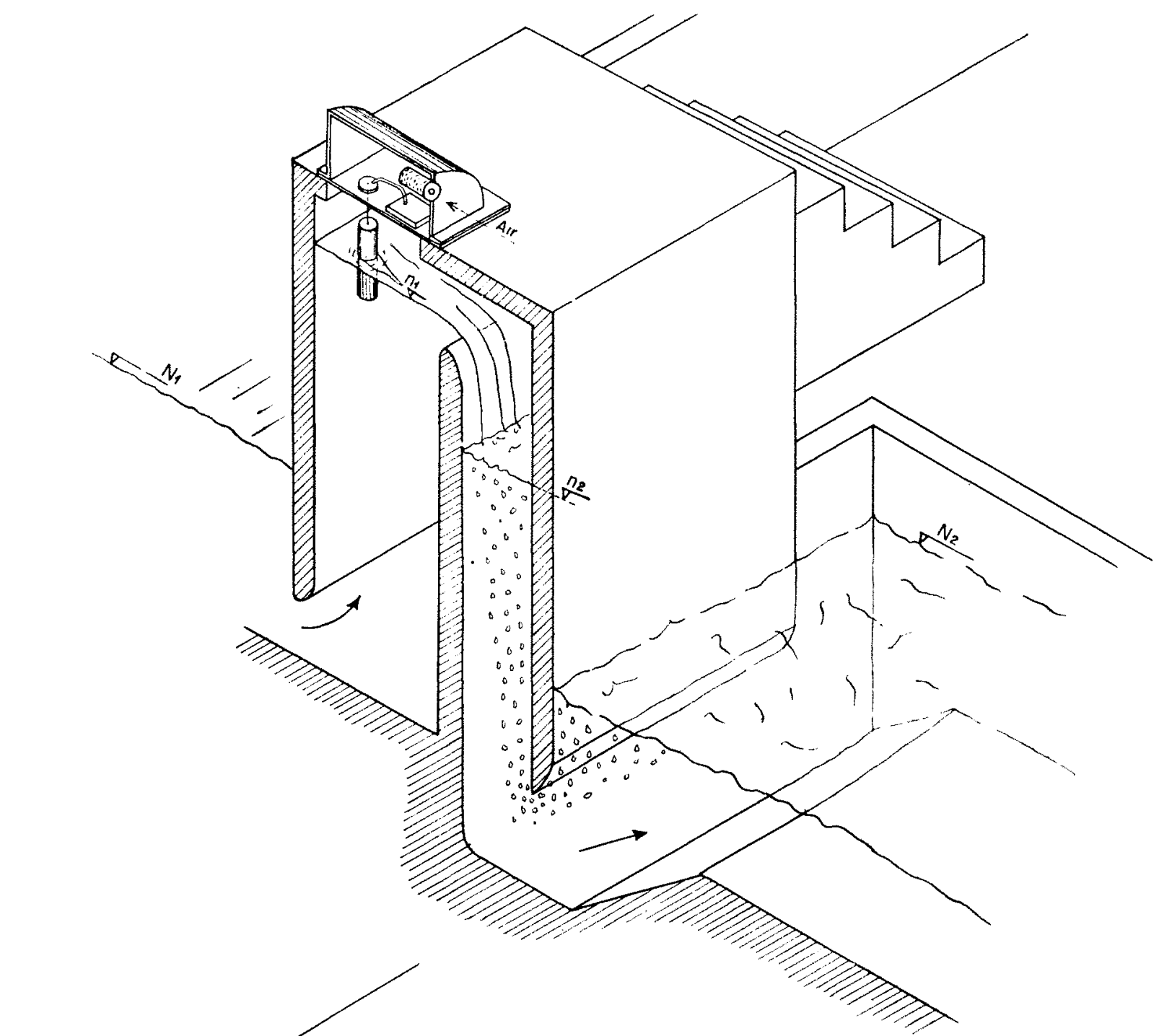

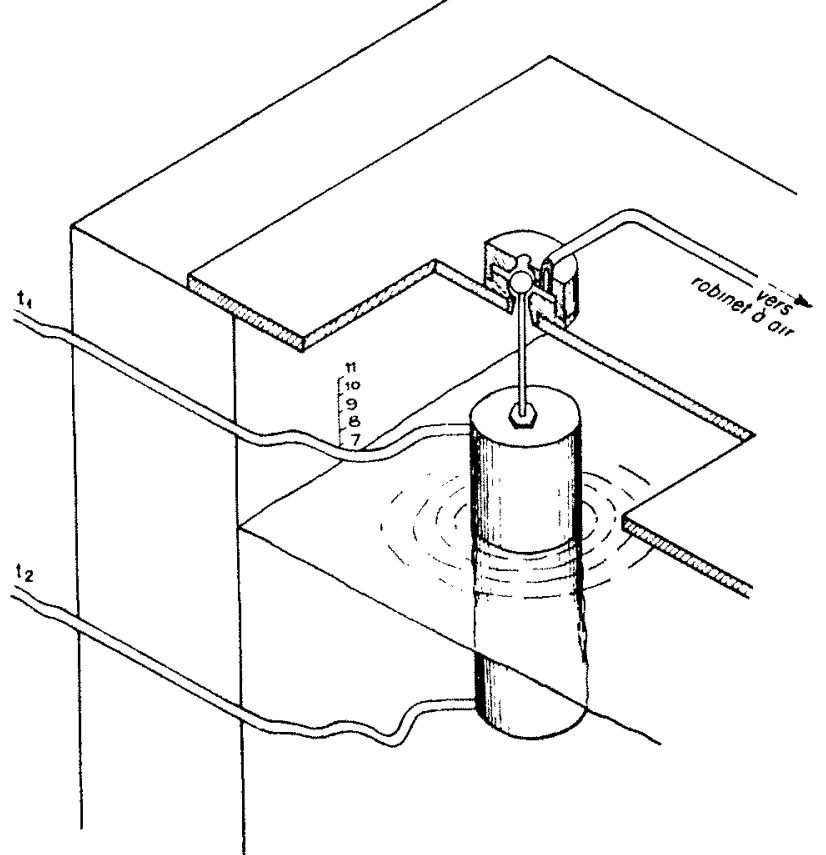

FIa, 2.

FIg. 1.

faciliter la mise en route qui demande un amorçage préalable.

- Un filtre à air grossier doit être placé à l'amont des organes contrôlant la circulation d'air afin d'assurer le bon fonctionnement de ces derniers. L'appareillage pneumatique qui fait partie du même domaine que les freins de chemin de fer, par exemple, sera, de cette facon, sans défaillance et ne nécessitera pratiquement pas d'entretien particulier.

Si les remarques précédentes font penser à des inconvénients qui resteront sans gravité, on voudra bien considérer les avantages obtenus et qui sont les suivants :

- L'appareil est simple et robuste en ce sens qu'il est en grande partie statique et que le réglage du niveau se fait presque sans toucher l'eau.

- L'eau déversante et le flotteur sont à l'abri de tout, y compris du gel. 


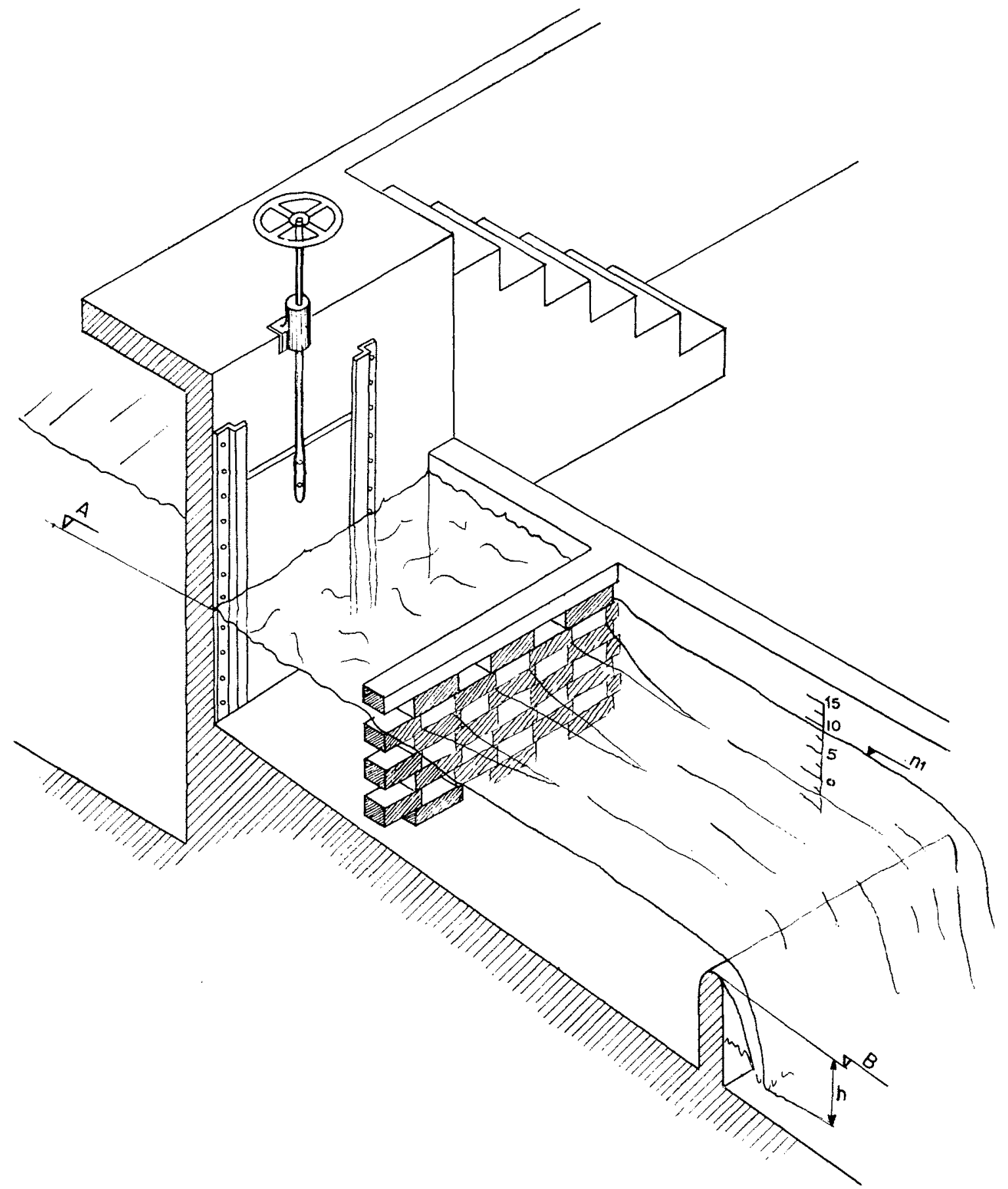

Fra. 3.

-- Les variations lentes des niveaux amont et aval n'ont pas d'influence sur le réglage du niveau $n_{1}$.

- La délermination du niveau d'arasement du déversoir est simple, il suffit que celui-ci soit nettement plus haut que le niveau amonl maximum.

- Le niveau amont minimum $\mathrm{N}_{1}$ n'est pas défini a priori puisqu'il peut s'abaisser d'autant plus que le niveau aval $N_{2}$ de restitution s'abaisse lui-mème. Le niveau minimum amont de fonctionnement est simplement plus haut que le niveau aval de la valeur de la perte de charge nécessaire.

Cette possibilité n'existe pas avec des dispositifs couramment employẻs (fig. 3) constitués d'une vanne (réglant un niveau aval $n_{1}$ ), suivie d'un déversoir. Dans ce dernier cas, en effet, c'est le niveau maximum possible $B$ de l'eau à l'aval du déversoir à l'air libre qui impose l'arasement de ce dernier; si Je niveau aval baisse, il apparaît une chute plus grande à l'aval du 
déversoir, cette chute $h$ constitue une perte de charge supplémentaire irrécupérable. Pour une installation donnće, basće sur le principe de la figure 3 , le niveau amont minimum $\mathrm{A}$ et le niveau aval maximum $B$ sont $\dot{a}$ des cotes fixées en valeur absolue.

Avec le module hydropneumatique, tout report de charge de l'aval vers l'amont est rendu continuellement possible à travers l'appareil parce que le déversoir est en atmosphère déprimée. Seul un orifice débitant sous le niveau aval donnerait un effet analogue.

- La perte de charge minimum demandée par l'appareil est d'ailleurs très faible parce que le déversoir est pratiquement le seul demandant une charge pour fonctionner.

La perte de charge de la vanne représentée figure 3 , et celle du tranquillisateur, ont disparu sur la figure 1 en même temps que la vanne et le tranquillisateur.

Le module hydropneumatique n'est donc pas le siège de pertes de charge en cascades.

- C'est un déversoir qui mesure lc débit et non un orifice. Or le déversoir est bien plus précis pour le réglage des débits moýens ou faibles lorsque la précision de réglage du niveau $n_{1}$ est constante en valeur absolue.

Si l'on avait choisi un orifice, c'est une différence de pression qu'il eût mieux valu régler.

Avec le déversoir, c'est un niveau constant qu'il suffit d'obtenir, niveau que l'on rìgle, avec une précison de l'ordre du millimètre si on le désire.

- Le contrôle du débit a licu d'abord et la libération d’énergie excédentaire a lieu ensuite (contrairement à l'exemple de la figure 3 où une vanne de réglaşe précède le déversoir de contrôle).

Le module hydropneumatique permel done de mesurer et de contrôler le débit d'une façon parfaite en un point d'où sont éliminés les tourbillons qui suivent toul organe de dissipalion d'énergie.
Les dispositifs de tranquillisation sont en mème temps économisés et l'énergic excédentaire participe, de son côté, à l'entrelien de la marche de l'appareil.

Conclusron. - L'appareil à débit constant réglable dénommé « module hydropneumatique » n'est assurément pas universel, ne serait-ce que parce qu'il ne saurait admettre des variations du niveau amont de plus de 8 à $9 \mathrm{~m}$ : on atteindrait en effet la limite de vaporisation de l'eau.

Il a été créé à l'origine pour équiper la sortie de grands filtres. Pour ce genre de problème, les avantages suivants (dont l'existence a été prouvée par le fonctionnement continu d'un prototype industriel) ont motivé l'intérêt porté à un tel régulateur :

- L'appareil est robuste et ses réglages sont précis;

- Des débits relativement importanls $(240 \mathrm{l} / \mathrm{s}$ sur le prototype mentionné) sont facilement obtenus;

- Ia perte de charge est minime si on le dísire ( $5 \mathrm{~cm}$ env.);

- Une télécommande éventuelle est facile à installer, simple, souple et précise;

- Les réponses sont rapides;

-- La charge récupérée à l'aval peut être reportée à l'amont, c'est-à-dire sur les filtres;

- Ia hotte constitue un ahri ris-à-vis du gel;

-- Le prix de l'ensemble est réduit si on le compare à celui d'autres types d'appareils.

Mais on peut assurément précoir un certain nombre d'applications à d'autres domaines. Citons, par exemple, les problimes de restitution d'un débit constant à l'aval d'un harrage, les problemes de distribution dans les réscaux d'irrigation, certains problèmes d'égouts.

En ces derniers domaines où la précision demandéc pourrait être, en principe, moindre, une réalisation plus simple encore est concevable. Les créateurs de l'appareil seraient assurément disposés à collaborer avee toule personne qu’une application quelconque de ce rigulateur de dibil intéresserait.

\section{II. - LE TIREUR D'AIR}

Une application très particulière, et peut-être inattendue, du module précédent, est le tireur d'air, une sorte d'éjecteur fonctionnant avec de l'eau sous une charge totale extrêmement faible et créant une dépression très nettement plus grande, susceptible d'entraîner de l'air comme le ferait une pompe à vide.

Il s'agit d'un véritable siphon comportant. comme dans le cas précédent, un flotteur réglant un niveau d'eau intérieur et actionnant un robi- net d'air. Contrairement au cas décrit précédemment (et paree que la précision du réglage n'a pas d'importance), le flotteur altaque directement le robinet qui est un pointeau, l'air aspiré vient d'une capacité où l'on veut faire le vide; par ailleurs, il ne s'agit plus d'un deversemenl, mais d'un écoulement en puits sature.

Le siphon sera avantageusement composé de deux tubes concentriques (fig. 4), le tube intérieur formant la branche aval sera terminé vers 
le haut par un évasement. Le flotteur sera annulaire pour ne pas gêner le vortex apparaissant au centre; l'air déprimé enfermé en haut sous la hotte passera par le centre de ce vortex pour se mélanger à l'eau descendante. Cet appauvrissement en air sous la hotte fera monter le niveau de l'eau, et, par l'intermédiaire du flotteur, pro-

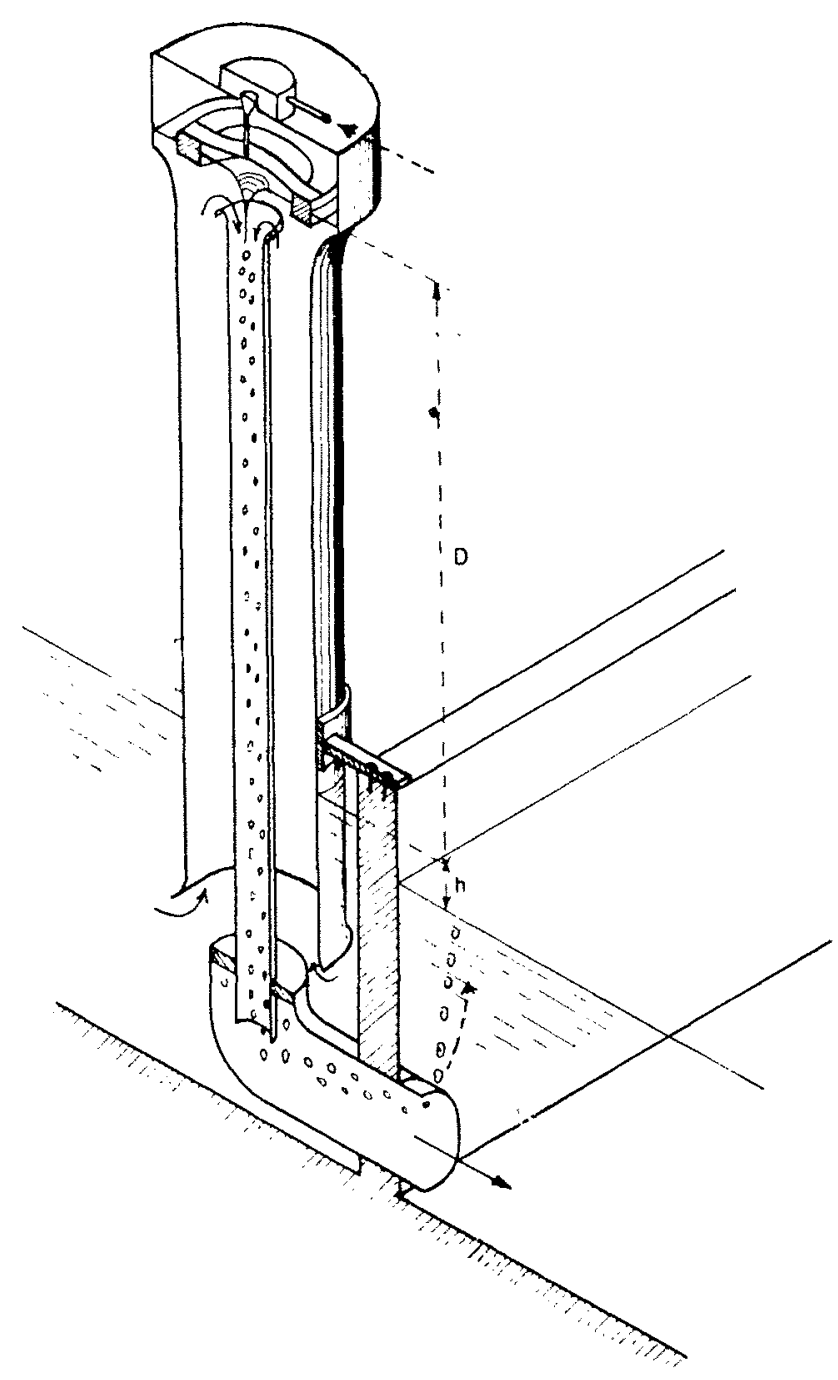

F1c. 4.

voquera l'ouverture du pointeau qui réapprovisionnera la poche d'air tout en empêchant le désamorçage du siphon.
On constate que la dépression $D$ est obtenue à l'aíde d'un phénomène pratiquement statique : on fait monter l'eau pour que, au sommet de l'appareil, elle soit à la dépression désirée. Isa petite charge motrice nécessaire $h$ servira seulement à lutter contre les pertes de charge des tubes, à créer un V2/2 $g$ au col de la branche descendante pour permettre à l'air sous la holte de pénétrer dans l'eau, à produire une vitesse dè quelque $50 \mathrm{~cm} / \mathrm{s}$ dans la branche descendante pour que les bulles d'air soient entraînées vers le bas, à lutter contre la différence de densité de l'enu dans la branche montante et du mélange air et eau dans la branche descendante.

Il n'y a pas de commune mesure entre la charge motrice, qui peut descendre a 4 ou $5 \mathrm{~cm} / \mathrm{s}$ (nous l'avons vérifié sur un appareil ayant une branche descendante de $125 \mathrm{~mm}$ de diamètre) et la dépression qui, théoriquement, peut atteindre $8 \mathrm{~m}$, semble-t-il, avec un appareil de hauteur correspondante. Dans un tel cas extrême, le rapport $\mathrm{D} / \mathrm{h}$ serait de près de 200 .

On comparera ce chiffre tout d'abord avec celui des venturis où le rapport dépression/charge ne dépasse pas 10 , puisque le rendement du divergent ne peut dépasser $90 \%$. Mais si. du venturi, on passe à l'éjecteur courant où le rendement s'effondre en raison du mélange des fluides moteur et entrainé, le même rapport $\mathrm{D} / \mathrm{h}$ est re l'ordre de 3 à 5 seulement.

En résumé, nous avons affaire à un éjecteur très schématisé monté au sommet d'un siphon dont la partialisation est contrôlée par un équipage mobile simplifié.

Le rapport $\mathrm{D} / h$ passe des chiffres habituels de 3 ou 5 à 200 . Le rendement est. par conséquent, très nettement amélioré; de plus, on peuit envisager des charges motrices remarquablement faibles si l'on n'a pas mieux à mettre à la disposition de ce tireur d'air que nous présentons à titre de curiosité.

Signalons d'ailleurs, pour terminer, que si le débit d'air augmente avec la charge motrice, le débit d'eau, lui, ne varie pas. En nous excusant de leur livrer un problème trop facile, nous laissons aux disciples du professeur Cyprien LEBORGNE le soin de trouver si ce tireur d'air est aussi un appareil à débit constant, ou, le cas échẻant, quelles sont les améliorations à lui apporter pour qu'il le soit. 


\section{III. - LE SIPHON “BÉNITIER"}

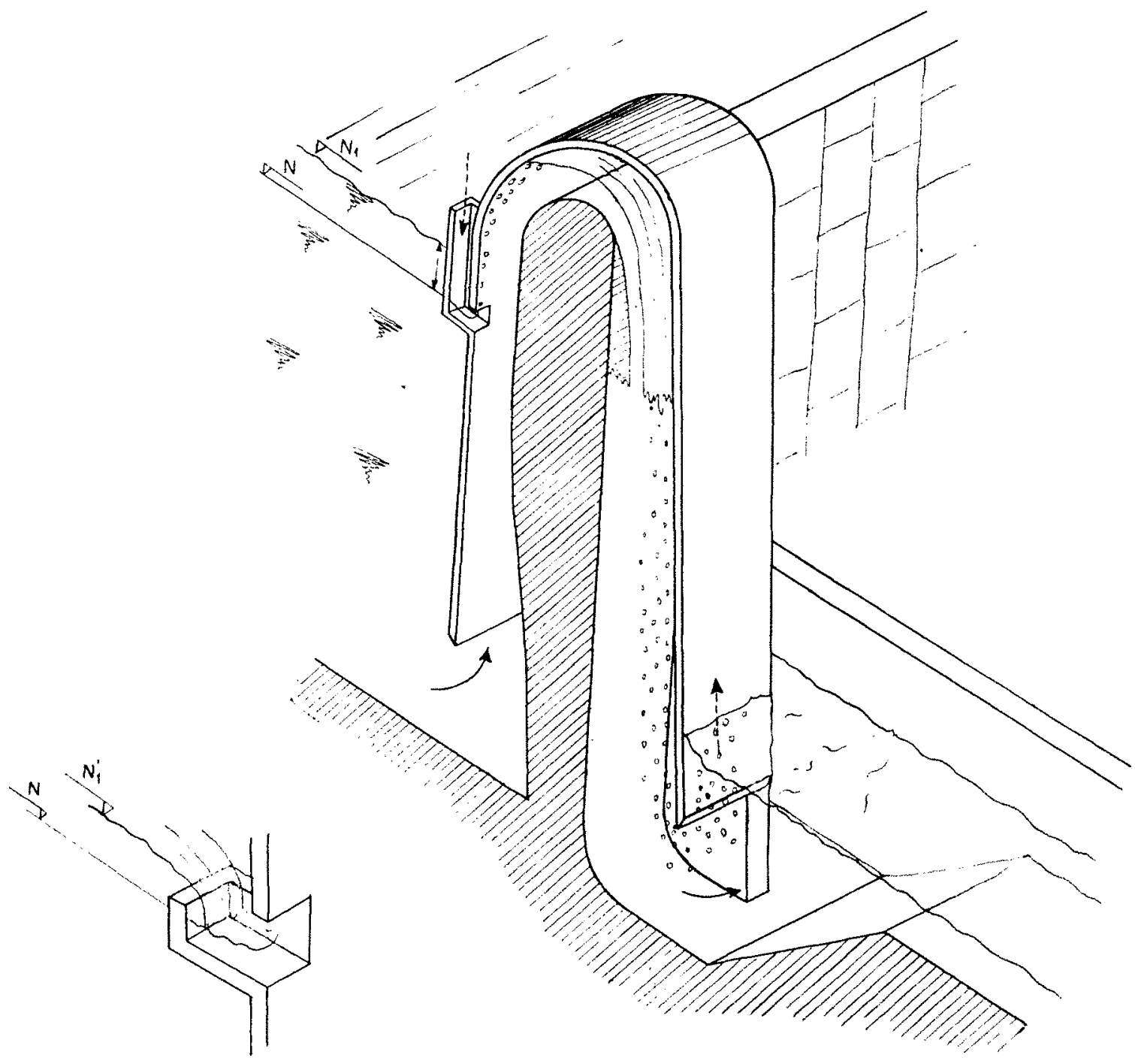

ivic. 5 bis.

F1c. 5.

La limitation d'un niveau ḋ une altitude maximum peut être obtenue, par exemple, avec un déversoir (trop-plein ou évacuateur de crue), un siphon, un clapet ou une vanne automatique à niveau amont constant.

Si l'on choisit un siphon, il sera nécessaire de l'équiper d'une façon particulière pour lui permettre de régler son débit à des valeurs partielles correspondant au débit excédentaire du moment; une simple tubulure destinée à introduire de l'air dans la hotte pour assurer un désamorçage partiel constituera souvent cel equipement. 


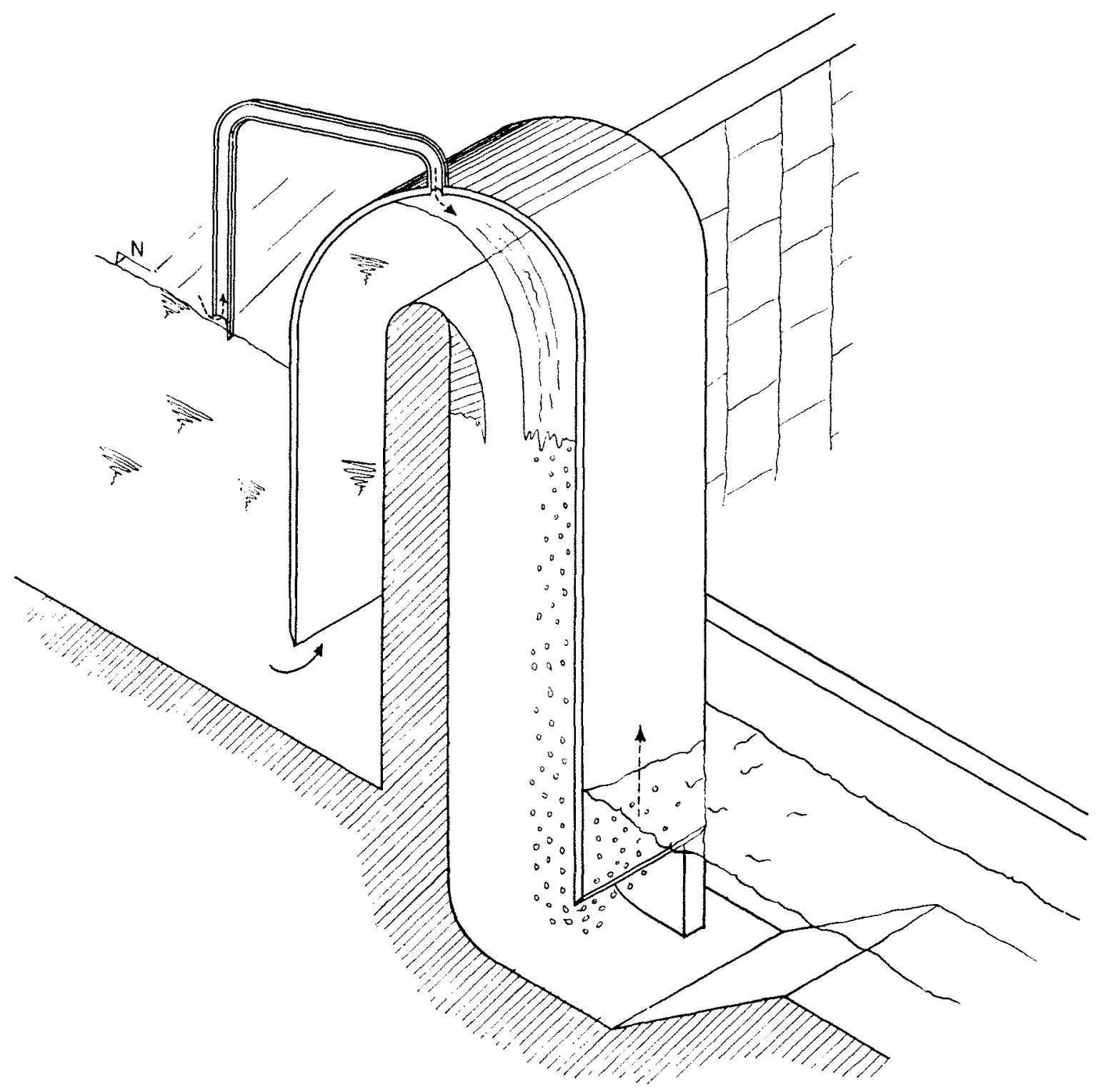

FIG. 6.

Nous rappellerons tout d'abord pourquoi il est relativement facile d'obtenir le fonctionnement partialisé d'un siphon dans les limites de $1 / 3$ à $100 / 100$ du débit d'eau maximum pour lequel est fait l'appareil, pourquoi il est difficile de descendre au-dessous de $1 / 3$ et comment nous sommes arrivés à $1 / 12$ sans aucune complication matérielle.

\section{$*$}

Le siphon partialisé classique représenté sur la figure 6 peut fonctionner aux débits partiels par introduction d'air à l'amont du tuyau $\mathrm{T}$ obturé plus ou moins par le plan d'eau amont $\mathrm{N}$ que l'on cherche à régler. L'air intérieur est continuellement évacué à l'aval sous l'effet de la chute interne et, en régime continu, on doit avoir l'égalité du débit d'air entrant et du débit d'air sortant. Or, à débit d'eau nul, il n'y a pas de débit d'air sortant, et à plein débit d'eau il ne doit pas y avoir d'air entrant; le niveau amont réglé doit donc obturer l'orifice de partialisation aussi bien à débit nul qu'à plein débit et le découvrir partiellement aux débits d'eau intermé- 
diaires; pour l'un d'eux (et toutes choses égales d'ailleurs) existe un maximum de débit d'air.

La figure 7 représente la relation entre le niveau amont réglé $\mathrm{N}$ et de débit d'eau $\mathrm{Q}$; on concoit que, dans la partie descendante de la courbe $\mathbf{N}$, les régimes stables ne puissent être obtenus (décrément négatif). On considère habituellement que le point bas de la courbe est situé aux environs du $1 / 3 \mathrm{du}$ débit maximum et c'est pour cela que toute une zone de débits d'eau allant de 0 au $1 / 3$ ne pourrait pas être réglée d'une façon stable avec l'appareillage pris en exemple.

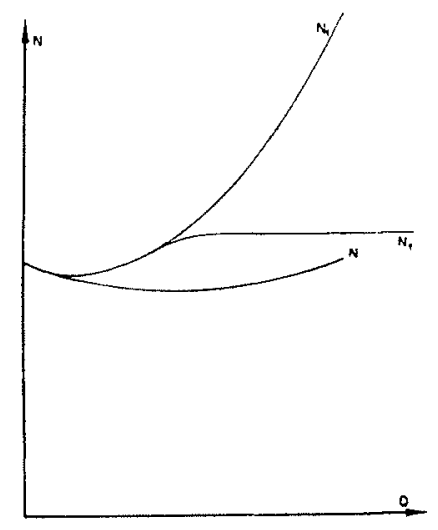

FIG. 7.

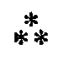

Dans le siphon «BéNitier », dit aussi «autostable $\gg$ et faisant l'objet de cette note, d'une part la tubulure T est supprimée (fig. 5) et remplacée par une lumière $O$ percée directement dans la paroi amont du siphon, d'autre part le niveau $\mathbf{N}$, obturant l'orifice, subit obligaloirement les mêmes variations que précédemment, mais ce n'est pas le niveau amont réglé.

Le niveau amont réglé $N_{1}$ est à l'extérieur et à l'amont d'une coupelle placée en regard et à l'amont de l'orifice $O$. Cette coupelle fait apparaître unc différence

$\mathrm{N}_{1}-\mathrm{N}=\mathrm{V}^{2} / 2 g+$ perte de charge à l'entrée, où $V$ est la vitesse de l'eau dans la branche ascendante à proximité de l'orifice : vitesse que l'on exagère par exemple à l'aide d'un rétrécissement de la branche amont dans une zone avoisinant le niveau $\mathrm{N}$.

La relation entre $\mathrm{N}_{1}$ et le débit d'eau $Q$, reportée sur la figure 7 , a une partie descendante qui correspond encore à des régimes instables mais dans une plage beaucoup plus réduite que précédemment, et réduite d'autant plus qu'on aura exagéré le rétrécissement pour augmenter la vitesse $V$. Sur un modèle qui a donné lieu à des réalisations dans plusieurs pays, nous avons obtenu sans difficulté des réglages entre $1 / 12$ et $100 / 100$ du débit total.

II faut remarquer sans tarder que cette courbe $\mathrm{N}_{1}$ de la figure 7 est loin d'être une horizontale et que le niveau amont réglé serait loin d'être quasi constant sans autre artifice.

Pour obtenir cette quasi-constance el modifier encore une fois la courbe d'origine, il suffit de supprimer le bénitier dès l'instant où l'on a "passé le cap » de la zone des débits d'ean difficiles à régler, e'est-à-dire de limiter le bénétier en hauteur (fig. 5 bis) pour que l'eau amont déverse par-dessus le bord de celui-ci, et le «court-circuite» dés que le débit $Q$ atteint une certaine valeur; ceci étant réalisé, une nouvelle courbe $N_{1}$ apparaît sur la figure 7 , elle pourra être considérée comme étant suffisamment horizontale pour un certain nombre d'applications pratiques.

$$
* *
$$

Voici done un siphon à niveau amont quasi constant qui pourrait, il est vrai, suggérer d'autres remarques d'ailleurs faciles.

Résumons nous en disant que par le choix de proportions peut-être assez parliculières dans les sections, par la position et la forme de l'orifice d'entréc d'air, enfin, par l'emploi du « bénitier », toutes choses extrêmement simples, il semble que l'on puisse obtenir, d'un type d'appareil deja couramment employé, un service plus régulier ct plus complet.

$$
\text { *** }
$$

Ce sera le dernier des siphons constituant le triptyque présenté ici. 